\title{
TonB-dependent receptor epitopes expressed in M. bovis BCG induced significant protection in the hamster model of leptospirosis
}

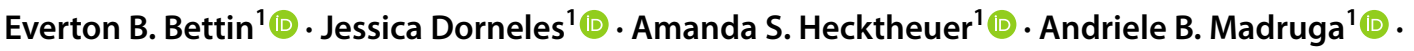

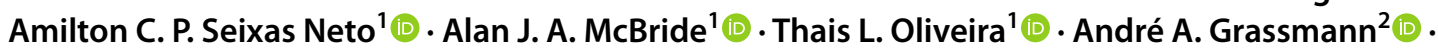 \\ Odir A. Dellagostin ${ }^{1}$ (D)
}

Received: 12 October 2021 / Revised: 26 October 2021 / Accepted: 30 November 2021 / Published online: 11 December 2021 (c) The Author(s), under exclusive licence to Springer-Verlag GmbH Germany, part of Springer Nature 2021

\begin{abstract}
Leptospirosis is an emerging infectious disease caused by pathogenic Leptospira spp. A universal vaccine against leptospirosis is likely to require highly conserved epitopes from pathogenic leptospires that are exposed on the bacterial surface and that generate a protective and sterilizing immune response. Our group recently identified several genes predicted to encode TonB-dependent receptors (TBDR) in Leptospira interrogans using a reverse vaccinology approach. Three leptospiral TBDRs were previously described and partially characterized as ferric-citrate, hemin, and cobalamin transporters. In the current study, we designed a fusion protein composed of predicted surface-exposed epitopes from three conserved leptospiral TBDRs. Based on their three-dimensional structural models and the prediction of immunogenic regions, nine putative surface-exposed fragments were selected to compose a recombinant chimeric protein. A Mycobacterium bovis BCG strain expressing this chimeric antigen encoded in the $\mathrm{pUP} 500 / \mathrm{P}_{\mathrm{pAN}}$ mycobacterial expression vector was used to immunize Syrian hamsters. All animals (20/20) vaccinated with recombinant BCG survived infection with an endpoint dose of $L$. interrogans $(p<0.001)$. No animal survived in the negative control group. Immunization with our recombinant BCG elicited a humoral immune response against leptospiral TBDRs, as demonstrated by ELISA and immunoblot. No leptospiral DNA was detected by lipL32 qPCR in the kidneys of vaccinated hamsters. Similarly, no growth was observed in macerated kidney cultures from the same animals, suggesting the induction of a sterilizing immune response. Design of new vaccine antigens based on the structure of outer membrane proteins is a promising approach to overcome the impact of leptospirosis by vaccination.
\end{abstract}

\section{Key points}

- Predicted surface-exposed epitopes were identified in three leptospiral TBDRs.

- An M. bovis BCG strain expressing a chimeric protein (rTBDRchi) was constructed.

- Hamsters vaccinated with rBCG:TBDRchi were protected from lethal leptospirosis.

Keywords Leptospira $\cdot$ Reverse and structural vaccinology $\cdot$ Beta-barrel transmembrane protein · Epitope-based vaccines . Chimeric protein

\section{Introduction}

Leptospirosis is a major zoonosis worldwide in terms of morbidity and mortality rates. The global incidence is estimated to be over one million cases every year, resulting in $\sim 60,000$

Everton B Bettin and Jessica Dorneles contributed equally.

\section{Everton B. Bettin}

1 Centro de Desenvolvimento Tecnológico, Unidade de Biotecnologia, Universidade Federal de Pelotas, Pelotas, RS, Brazil

2 Department of Medicine, University of Connecticut Health, Farmington, CT, USA deaths (Costa et al. 2015). Rodents are the primary reservoir hosts of pathogenic Leptospira spp., while humans are incidental hosts. The disease also affects several wild and domestic animals (Ellis 2015; Hake and Levett 2015). Leptospirosis in humans can vary from mild symptoms, such as fever and myalgia to severe outcomes, with the development of acute kidney injury and pulmonary hemorrhage syndrome (Haake and Levett 2015). Leptospiral vaccines currently available are inactivated whole-cell preparations (bacterins) with widespread veterinary applications, but limited use in humans. Bacterins confer a short-term immune response that is protective only against the serovars included in the vaccine formulation (Adler 2015). Several recombinant proteins 
have been evaluated towards the development of new and improved leptospiral vaccines, with variable success (Felix et al. 2020). While some experimental recombinant vaccines were protective against lethal challenge, the majority failed to achieve heterologous and/or sterilizing protection, highlighting the need to evaluate novel vaccination strategies (Grassmann et al. 2017b; Felix et al. 2020). A new and effective vaccine against leptospirosis will likely include surfaceexposed epitopes conserved among pathogenic Leptospira spp., allowing bacterial recognition by humoral immune response (Grassmann et al. 2017a, b; Dellagostin et al. 2017). Our group previously used a reverse and structural vaccinology approach to identify leptospiral beta-barrel outer membrane proteins ( $\beta b$-OMP), including six TonB-dependent receptors (TBDR) (Grassmann et al. 2017a).

TBDRs are outer-membrane proteins that promote the uptake of essential nutrients such as iron, nickel, vitamins, and carbohydrates (Noinaj et al. 2010). Since iron has limited bioavailability, its acquisition by bacteria is facilitated by the formation of complexes with other transport molecules, such as heme and siderophores, which are then internalized by specific TonB-dependent transport systems (Noinaj et al. 2010). Pathogenic and saprophytic Leptospira spp. require iron for in vitro growth (Faine 1959) and evolved mechanisms for iron sensing, acquisition, and utilization (Louvel et al. 2006). Two leptospiral TBDRs, encoded by LIC10896 and LIC10964, were described as homologs to the ferric citrate transporter FecA and the PhuR hemin receptor (Nascimento et al. 2004; Louvel et al. 2006), respectively. Another TBDR, LIC12374, is annotated as a homolog of the cobalamin transporter BtuB (Nascimento et al. 2004; Louvel et al. 2006). Cobalamin (or vitamin B12) is a cofactor for enzymes related to a variety of essential functions, including those related to carbon metabolism and biosynthesis of nucleotides (Shelton et al. 2019). Similarly to iron, cobalamin is a small nutrient required for Leptospira spp. survival (Louvel et al. 2006). While saprophytic species rely on external sources, pathogenic leptospires encode a pathway for de novo production of cobalamin from L-glutamate (Fouts et al. 2016). Due to their important role in bacterial metabolism and nutrient acquisition, several TBDRs have been evaluated as vaccine candidates against many pathogens, resulting in successful protection (Alteri et al. 2009; Hu et al. 2012; Hubert et al. 2013).

Epitope mapping in vaccine candidates permits the identification of regions with increased probability to elicit an immune response, consequently improving the antigenicity and immunogenicity of vaccine formulations and avoiding production of undesirable or ineffective responses (Oyarzún and Kobe 2016; Parvizpour et al. 2020). The rational selection of conserved epitopes also contributes to cross-protection
(Liu et al. 2017; Nosrati et al. 2019; Xiao et al. 2020), a particularly important aspect to consider for the development of leptospirosis vaccines, where the achievements of heterologous, long-term protection are major goals. Combined with protein structural information, epitope selection is a powerful tool to identify immunogenic surface-exposed fragments required for bacterial opsonization (Grassmann et al. 2017a). Opsonization of leptospires by specific immunoglobulins improved bacterial phagocytosis and clearance (GomesSolecki et al. 2017; Santecchia et al. 2020). Even though the immune response required for protection against leptospirosis is not yet fully understood, induction of a balanced cellular/ humoral response is believed to be key for a protective immune response (Gomes-Solecki et al. 2017; Grassmann et al. 2017b; da Cunha et al. 2019).

Mycobacterium bovis Bacillus Calmette-Guérin (BCG) is an attenuated strain widely used as a vaccine against human tuberculosis (TB) (Dockrell and Smith 2017). In use for a hundred years, BCG vaccination is a highly cost-effective intervention against tuberculosis and is recommended for all newborns in countries with a high burden of TB (Trunz et al. 2006; Lobo et al. 2021). BCG triggers a long-lasting immunity after a single dose, presenting several adjuvant properties and a strong cellular response (Dockrell and Smith 2017). BCG-induced adaptative immune response include the expression of several interleukins, such as TNF- $\alpha$ and IFN- $\gamma$, and, consequently, the proliferation and activation of macrophages (Dockrell and Smith 2017; Covián et al. 2019). Several studies have demonstrated the potential of recombinant BCG (rBCG) to enhance the immune response towards heterologous antigens (Gröschel et al. 2017; Soto et al. 2018), including those from $L$. interrogans (Seixas et al. 2007; Oliveira et al. 2019a; Dorneles et al. 2020), and represents a promising alternative for antigen delivery (Oliveira et al. 2017).

In this study, we identified highly conserved surfaceexposed regions containing $\mathrm{B}$ and $\mathrm{T} \mathrm{CD} 4+$ cell epitopes in three leptospiral TBDRs: LIC10896, LIC10964, and LIC12374. These fragments were combined on a multi-epitope fusion protein and evaluated using BCG as a vectorized vaccine carrier. Our preparation induced a protective immune response in the hamster model of acute leptospirosis, generating specific antibodies and sterilizing immunity.

\section{Materials and methods}

\section{Bacterial strains and growth conditions}

Escherichia coli strains BL21 (DE3) Star and TOP10 (Invitrogen, São Paulo, SP, Brazil) were grown at $37{ }^{\circ} \mathrm{C}$ in Lysogeny Broth (LB) medium, with $100 \mu \mathrm{g} \cdot \mathrm{ml}^{-1}$ ampicillin when required. M. bovis BCG (Pasteur and recombinant 
strains) were cultivated at $37{ }^{\circ} \mathrm{C}$ in Middlebrook $7 \mathrm{H} 9$ broth (Difco, BD, São Paulo, SP, Brazil) supplemented with $0.5 \%$ glycerol; $0.05 \%$ Tween 80 and $10 \%$ Oleic acid, Albumin, Dextrose Complex (OADC); or in 7H10 agar (Difco, BD, São Paulo, SP, Brazil) with 10\% OADC. The BCG media was supplemented with kanamycin, $25 \mu \mathrm{g}$. $\mathrm{ml}^{-1}$, when needed. Low-passage virulent $L$. interrogans serogroup Icterohaemorrhagiae serovar (sv.) Copenhageni strain (st.) Fiocruz L1-130 (WDCM1012) was cultivated at $30{ }^{\circ} \mathrm{C}$ in Ellinghausen-McCullough-Johnson-Harris (EMJH) medium supplemented with Leptospira EMJH enrichment (Difco, BD, São Paulo, SP, Brazil).

\section{In silico structure predictions and epitope selection}

Three proteins (LIC10896, LIC10964, and LIC12374) from L. interrogans sv. Copenhageni st. Fiocruz L1-130 (Taxid:267,671), previously identified as leptospiral TonBDependent receptors, were selected for evaluation in this study. Three-dimensional (3D) structures were predicted by threading using the I-TASSER server (Yang and Zhang 2015). Models with the highest $\mathrm{C}$-score were selected for epitope prediction. The 3D protein structures were visualized using USCF Chimera 1.12 (Pettersen et al. 2004). Sequences for the CD4 + T cell epitopes, with high affinity to 14 different human major histocompatibility complex class II (MHC-II) alleles, were predicted using NetMHCII 2.2 (Nielsen and Lund 2009) at the default settings. For linear (continuous) B cell epitope prediction, amino acid sequences were analyzed using BepiPred 1.0 (Larsen et al. 2006). Only T cell epitopes with a strong binding affinity $($ IC50 $<50 \mathrm{nM})$ and amino acid residues with a score higher than 0.35 in the B cell epitope analysis were considered. Epitopes were manually mapped onto the predicted 3D models of each $\beta \mathrm{b}$-OMP in USCF Chimera 1.12. These models were aligned with their best structural template obtained from the Orientations of Proteins in Membranes (OPM) database (Lomize et al. 2012). All surface-exposed fragments containing B and T CD4 + cell epitopes were used for antigen construction. Orthologs for each L. interrogans TBDR were identified by BlastP (NCBI) in 10 pathogenic (P1 clade) Leptospira spp. proteomes (Online Resource 1, Table S1). Protein sequences with $>70 \%$ identity and $40 \%$ query coverage were considered orthologs. Identity between the $L$. interrogans polypeptides and their orthologs was determined after multiple sequence alignments (MSA) using MUSCLE (EMBL-EBI) (Edgar et al. 2004).

\section{Recombinant protein expression and purification}

Selected surface-exposed fragments were assembled in silico using Vector NTI Advance ${ }^{\circledR} 11.5$ (Invitrogen) without linkers, to construct the fusion proteins rTBDRchi, rLIC10896chi, rLIC10964chi, and rLIC12374chi (Online Resource 2). E. coli codon optimized coding sequences (CDS) for each fusion protein were synthesized by Epoch Life Science (Texas, USA) and cloned into E. coli expression vector pAE (Ramos et al. 2004) using BamHI and Hin$d$ III restriction sites. Recombinant plasmids were used to transform E. coli BL21 (DE3) C41 strain (Invitrogen, São Paulo, SP, Brazil) by heat shock. When cultures reached mid-log phase (0.6-0.8 absorbance at $\left.\mathrm{OD}_{600}\right)$, expression was induced by adding $0.5 \mathrm{mM}$ IPTG (isopropyl- $\beta$-Dthiogalactopyranoside) and incubated at $37^{\circ} \mathrm{C}$ with agitation. After $4 \mathrm{~h}$, cells were harvested by centrifugation $\left(7,000 \times g, 15 \mathrm{~min}, 4{ }^{\circ} \mathrm{C}\right)$. Pellets were washed in phosphate-buffered saline (PBS) and resuspended in lysis buffer ( $20 \mathrm{mM}$ sodium phosphate, $0.5 \mathrm{M} \mathrm{NaCl}$, and $20 \mathrm{mM}$ Imidazole, $\mathrm{pH}$ 8.0) before sonication with six 30 -s pulses on ice. After lysis, insoluble proteins were harvested by centrifugation $\left(10,000 \times g, 1 \mathrm{~h}, 4^{\circ} \mathrm{C}\right)$ and resuspended in denaturing binding buffer ( $8 \mathrm{M}$ urea, $20 \mathrm{mM}$ sodium phosphate, $0.5 \mathrm{M} \mathrm{NaCl}$, and $20 \mathrm{mM}$ Imidazole, $\mathrm{pH} 8,0$ ) and incubated under constant agitation for $16 \mathrm{~h}$. After a final centrifugation $\left(10,000 \times g, 1 \mathrm{~h}, 4^{\circ} \mathrm{C}\right)$, recombinant proteins were purified using the AKTA Start automated chromatography system (GE Healthcare). Briefly, the supernatant was applied to a nickel-charged HisTrap FF column (GE Healthcare, São Paulo, SP, Brazil), and after washing with 20 column volumes, recombinant fusion proteins were eluted over a total of $20 \mathrm{ml}$ using a $0-100 \%$ gradient of elution buffer $(8 \mathrm{M}$ urea, $20 \mathrm{mM}$ sodium phosphate, $0.5 \mathrm{M} \mathrm{NaCl}$, and $0.5 \mathrm{M} \mathrm{Imi-}$ dazole, $\mathrm{pH} 8,0)$. Purified proteins were then dialyzed against PBS at $4{ }^{\circ} \mathrm{C}$ for $24 \mathrm{~h}$ and stored at $-80{ }^{\circ} \mathrm{C}$ or $4{ }^{\circ} \mathrm{C}$.

\section{Production of anti-rTBDRchi hyperimmune sera}

Four-week-old female Wistar rats (Rattus norvegicus) were injected intraperitoneally with $50 \mu \mathrm{g}$ rTBDRchi emulsified in complete Freund adjuvant for the first dose and incomplete Freund adjuvant for the remaining two doses. Two weeks after the second boost, animals were euthanized by exsanguination, and hyperimmune sera were obtained by centrifugation $\left(3,500 \times g, 15 \mathrm{~min}, 4^{\circ} \mathrm{C}\right)$ and stored at $-20^{\circ} \mathrm{C}$.

\section{Construction of recombinant $M$. bovis BCG and expression analysis}

Using pAE/rTBDRchi as template, the rTBDRchi CDS was amplified by PCR using primers described in Online 
Resource 1 (Table S2). The PCR product was digested using $X b a \mathrm{I}$ and PstI restriction enzymes (New England BioLabs) and cloned into the BCG expression vector pUP500/PpAN (Oliveira et al. 2019b), previously digested with Spel e PstI (New England BioLabs), generating pUP500/PpAN:rTBDRchi plasmid. Recombinant pUP500/ PpAN:rTBDRchi was transformed into $M$. bovis BCG Pasteur cells by electroporation, as previously described (Parish and Stoker 1995). Cells were plated onto 7H10 agar (Difco, BD, São Paulo, SP, Brazil) containing $25 \mu \mathrm{g} \cdot \mathrm{ml}^{-1}$ of kanamycin and incubated at $37^{\circ} \mathrm{C}$ for 21 days. Resistant colonies were inoculated in selective Middlebrook 7H9 broth (Difco) and cultivated at $37{ }^{\circ} \mathrm{C}$ to evaluate recombinant expression by immunoblot. Briefly, whole-cell lysates were prepared from rBCG:TBDRchi grown to mid-logarithmic phase at $37{ }^{\circ} \mathrm{C}\left(\mathrm{OD}_{600}=0.8\right)$, when cells were collected by centrifugation $(4,000 \times g, 15 \mathrm{~min})$ and resuspended in Tris- $\mathrm{HCl}$ (pH 8), adjusting the cell density to $10^{7} \mathrm{CFU} \cdot \mathrm{ml}^{-1}$. rBCG:TBDRchi whole-cell lysates were separated in $12 \%$ polyacrylamide gels and transferred to nitrocellulose membranes (Hybond ECL, GE Healthcare, IL, USA). Membranes were incubated overnight with rat anti-rTBDRchi sera at 1:100 dilution, followed by anti-rat HRP-conjugated secondary antibodies (Sigma Aldrich, MO, USA) diluted 1:5,000. Immunoblots were developed using Pierce ECL Western Blotting Substrate (Thermo-Fisher, IL, USA).

\section{Vaccination and challenge of hamsters}

rBCG:TBDRchi and BCG Pasteur (negative control) strains were cultivated in vitro and collected by centrifugation $(4,000 \times g, 15 \mathrm{~min})$. Cells were resuspended in PBS, adjusting concentration to $10^{7}$ cells $/ \mathrm{ml}$ (considering $1 \mathrm{OD}_{600}=$ $1 \times 10^{8}$ cells $/ \mathrm{ml}$ ) and immediately used. Male Syrian hamsters (Mesocricetus auratus), 4-6 weeks old, were subcutaneously inoculated with $10^{6}$ cells of BCG Pasteur $(n=13$ per experiment) or recombinant BCG:TBDRchi ( $n=10$ per experiment). An additional control group ( $n=4$ per experiment) was immunized (intramuscular) with a leptospiral whole-cell bacterin $\left(10^{8}\right.$ heat-inactivated leptospires in $100 \mu \mathrm{l}$ PBS). Two doses of each formulation were administered 21 days apart. Thirty days after the second boost, hamsters were infected intraperitoneally with $10^{3}$ leptospires $(L$. interrogans sv. Copenhageni st. Fiocruz L1-130), equivalent to five times the average 50\% endpoint dose (ED50), as previously determined (Oliveira et al. 2019a). Hamsters were monitored daily for clinical signs of acute leptospirosis for 30 days after challenge. Criteria for humane euthanasia $\left(\mathrm{CO}_{2}\right.$ narcosis) was established as previously described (Coutinho et al. 2011) and includes the following: > 10\% weight loss, prostration, bristling, apathy, and lack of appetite. Blood samples were collected one day before the first immunization (day zero) and before challenge (day 51). A total of two independent experiments were performed. All animal procedures were conducted according to the rules and regulations of the Animal Experimentation Ethics Committee at UFPel.

\section{Analysis of the leptospiral burden in hamster kidneys post-challenge}

At the end of the experiment, kidneys were collected aseptically in order to evaluate the leptospiral burden postchallenge. Kidney samples were macerated in $10 \mathrm{ml}$ EMJH medium supplemented with $10 \%$ Leptospira enrichment supplement. After $1 \mathrm{~h}$ at $30{ }^{\circ} \mathrm{C}, 500 \mu \mathrm{l}$ of the macerate was inoculated into $10 \mathrm{ml}$ of fresh EMJH and incubated at $30^{\circ} \mathrm{C}$ for up to 8 weeks. Cultures were monitored weekly by darkfield microscopy. DNA extraction from kidney samples, followed by qPCR, was performed as previously described (Dorneles et al. 2020). Briefly, DNA was extracted from approximately $40 \mathrm{mg}$ of kidney tissue using SV Genomic DNA Purification Kit (Promega, Brazil) and quantified using Qubit 2.0 fluorometer (Thermo Fisher). Quantitative real-time PCR (qPCR) detection of leptospiral DNA was performed in a LightCycler 96 system (Roche, Basel, Switzerland), using $200 \mathrm{ng}$ of total DNA, $0.4 \mu \mathrm{M}$ of each primer specific to lipL32 (Online Resource 1, Table S2), and SYBR Green PCR Master Mix (Applied Biosystems, São Paulo, SP, Brazil). Reactions were performed in triplicate as previously described (Dorneles et al. 2020). A standard curve was generated from $10^{6}$ to 10 copies of lipL32 and samples were considered negative when their qPCR quantitation cycle $(\mathrm{Cq})$ was higher than that obtained for the lowest concentration of 10 copies. Absolute quantification analysis using LightCycler 96 software (Roche) was performed to determine the number of leptospire genome equivalents per reaction and thereafter converted to copies per $\mu \mathrm{g}$ of total DNA.

\section{Evaluation of the humoral immune response}

Humoral immune response elicited after vaccination was evaluated by indirect ELISA (enzyme-linked immunosorbent assay), as previously described (da Cunha et al. 2019). First, 96 well microtiter plates (Polysorp, Nunc, São Paulo, Brazil) were coated with $1 \mu \mathrm{g}$ of purified rTBDRchi per well at $4{ }^{\circ} \mathrm{C}$ overnight, in $0.1 \mathrm{M}$ carbonate-bicarbonate buffer, $\mathrm{pH}$ 9.6. Plates were washed three times with PBS- $0.05 \%$ Tween 20 (PBST) and then blocked with $200 \mu$ of $5 \%$ non-fat dry milk in PBS-T, for $1 \mathrm{~h}$ at $37^{\circ} \mathrm{C}$. Hamsters' sera were diluted 1:50 in PBST and evaluated in triplicate. After incubation for $1 \mathrm{~h}$ at $37^{\circ} \mathrm{C}$, plates were washed and HRP-conjugated anti-Syrian hamster IgG (Sigma Aldrich, MO, USA) was added at 1:5,000 dilution for $1 \mathrm{~h}$ at $37^{\circ} \mathrm{C}$. After a final wash, reactions were developed using o-phenylenediamine dihydrochloride (Sigma-Aldrich, MO, USA) and hydrogen 
peroxide and stopped after 15 min with $4 \mathrm{~N}_{2} \mathrm{SO}_{4}$. Absorbance was read at $492 \mathrm{~nm}$ wavelength in a microplate reader (Mindray MR-96A, São Paulo, Brazil).

Immunoblots were performed as previously described, with small changes (Groshong et al. 2021). Briefly, samples containing $1 \mu \mathrm{g}$ of each recombinant protein (rTBDRchi, rLIC10896, rLIC10964, and rLIC12374) or L. interrogans st. Fiocruz L1-130 whole-cell lysate $\left(10^{8} /\right.$ lane) were analyzed by SDS-PAGE and transferred to nitrocellulose membranes (GE Healthcare, IL, USA) at $20 \mathrm{~V}$ for 25 min using a semi-dry transfer (Bio-Rad). Membranes were blocked for $1 \mathrm{~h}$ at room temperature with $5 \%$ non-fat dried milk diluted in PBS-T and probed overnight at $4{ }^{\circ} \mathrm{C}$ with a pool of hamster sera obtained after immunization, at a 1:200 dilution in blocking buffer. After washing 5 times with PBS-T, membranes were incubated for $1 \mathrm{~h}$ at RT with an HRP-conjugated goat anti-hamster IgG antibody (Sigma Aldrich, MO, USA) diluted in blocking buffer $(1: 6,000)$. Following six washes with PBS-T, immunoblots were developed using the Pierce ECL Western Blotting Substrate (Thermo-Fisher, IL, USA) detection reagent, and results were visualized using C-Digit Blot Scanner (LI-COR, NE, USA).

\section{Statistical analysis}

Significant protection against lethal leptospirosis was evaluated using Fisher's exact test (two-tailed). Comparison of humoral immune response elicited by different experimental groups was performed using analysis of variance (one wayANOVA with Tukey multiple comparison). For all analyses, $p$ values $<0.05$ were considered significant. Graphical representations and statistical analysis were performed using GraphPad Prism 8.

\section{Results}

\section{Structural modeling, sequence analysis, and design of rTBDRchi}

Our group previously described leptospiral TonB-dependent receptor (TBDR) orthologs based on a reverse and structural vaccinology approach (Grassmann et al. 2017a). In this study, we selected the three TBDRs for evaluation as vaccine candidates against leptospirosis, LIC10896, LIC10964, and LIC12374, for which important nutrient transport functions were previously suggested (Nascimento et al. 2004; Louvel et al. 2006). The 3D models were generated using the standard threading assembly method by I-TASSER. All retrieved models with the best confidence score (C-score) were identified as structural analogs to TonB-dependent receptors with resolved structures, deposited on the Protein Data Bank (PDB) (Online Resource 1, Table S3). The I-TASSER server was also used to perform structural alignments between the leptospiral queries and their PDB templates using the TMalign program, with reported values varying from 0 to 1 , where 1 indicates a perfect superposition of two structures (Zhang and Skolnick 2005). Predicted structures for the leptospiral TBDRs LIC10896, LIC10964, and LIC12374 presented TM-Align values varying from 0.724 to 0.842 (Online Resource 1, Figure S1).

Next, we analyzed each of the selected leptospiral proteins in order to predict T CD4 + and linear B cell epitopes along their sequences. Several T CD $4+$ and linear B cell epitopes were identified along the full-length leptospiral TBDR sequences (Online Resource 3). Membrane-embed templates for each generated model were obtained from the Orientations of Proteins in Membranes (OPM) database (Lomize et al. 2012), and structural alignments were performed to identify the predicted surface-exposed fragments (Online Resource 1, Figure S1). Each identified T CD4 + and linear B cell epitope was mapped onto 3D structures allowing the selection of all predicted surfaceexposed regions containing antigenic determinants. The three TBDRs analyzed contained nine surface-exposed loops with $\mathrm{T}$ and B cell epitopes (Fig. 1a). Antigenic loops were selected and combined to construct a $41 \mathrm{kDa}$ recombinant protein, named rTBDRchi (Fig. 1b). Five fragments were selected from LIC10896 and two fragments from LIC10964 and LIC12374 (Fig. 1, Table 1). In silico approaches for the T CD4 + cell epitope prediction focused on the identification of core binding regions composed of nine residues (9-mer), which largely determined binding affinity and specificity for human leukocyte antigens (HLA) presentation (Sanchez-trincado et al. 2017). A total of 24 strong binder 9 -mers (IC50 $<50 \mathrm{nM}$ ) were identified in the exposed loops. Thirteen different HLA alleles (out of 14 alleles analyzed) were predicted to bind to the rTBDRchi epitopes (Online Resource 1, Table S4). rTBDRchi also included promiscuous $\mathrm{T}$ cell epitopes, with predicted binding to several HLAs, from all selected leptospiral TBDRs.

\section{rTBDRchi fragments are conserved among pathogenic Leptospira spp.}

The selected fragments were evaluated for amino acid conservation between $L$. interrogans and the orthologs from ten additional pathogenic (P1 clade) Leptospira spp. (Vincent et al. 2019). By performing a MSA, all sequences showed high conservation levels with identities varying from 73.7 to $100 \%$ for different rTBDRchi fragments (Online Resource 4). We were not able to identify a LIC 10896 ortholog in $L$. kmetyi, although fragments from LIC10964 and LIC12374 from L. interrogans were highly conserved (84.21-93.10\%) with their orthologs in L. kmetyi. As expected, due to their close phylogenetic relationship, L. kirschneri and $L$. 
Fig. 1 Epitope mapping of leptospiral TBDRs and in silico construction of rTBDRchi. a $\mathrm{CD} 4+\mathrm{T}$ cell epitopes and linear $B$ cell epitopes were identified by NetMHCII and BepiPred servers, respectively. Epitopes were mapped on 3D structures generated by I-TASSER. Surface-exposed fragments containing identified epitopes (colored) were used to construct the recombinant fusion protein. b Nine identified fragments were selected to construct a $41 \mathrm{kDa}$ recombinant fusion protein (rTBDRchi). rTBDRchi contains $11 \mathrm{CD} 4+\mathrm{T}$ cell (black dashes) and 10 linear B cell (blue dashes) epitopes throughout its sequence a
LIC10896

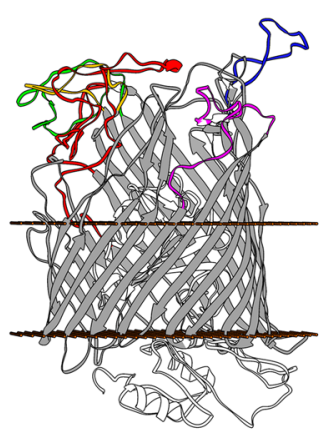

$\underbrace{}_{90^{\circ}}$

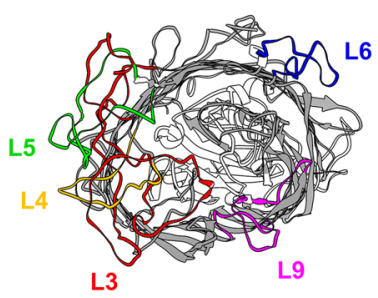

LIC10964

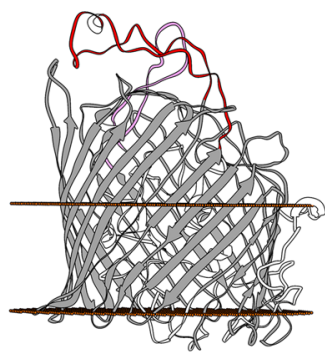

$\underbrace{}_{90^{\circ}}$

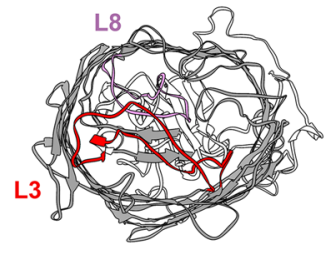

LIC12374

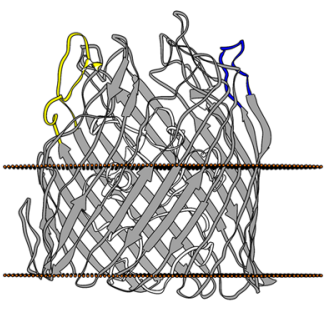

b

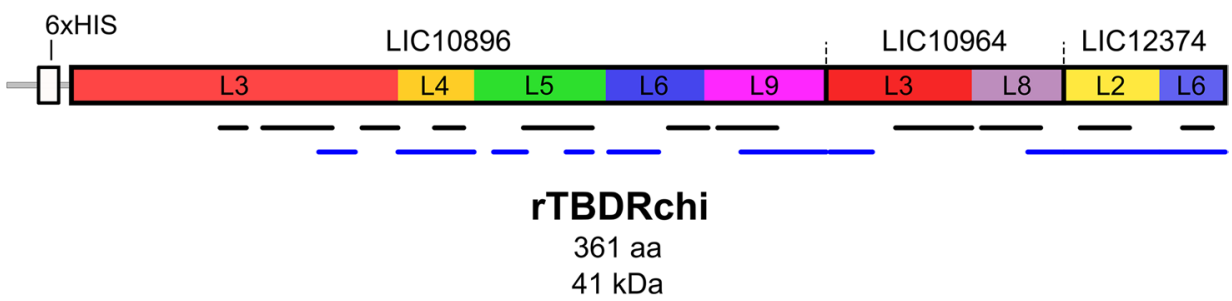

Table 1 Sequences of the selected fragments predicted to contain surface-exposed regions and $\mathrm{B}$ and $\mathrm{T}$ cell epitopes. Protein sequences were submitted to BepiPred and NetMHCII for prediction of linear B cell (bold) and T CD4 + cell (underlined) epitopes, respectively. All surface-exposed loops containing identified epitopes were selected to compose the fusion protein and are listed

\begin{tabular}{|c|c|c|}
\hline Protein & Fragment & Sequence \\
\hline \multirow[t]{5}{*}{$\underline{\mathrm{LIC} 10896}$} & $\underline{3}$ & $\begin{array}{l}\text { YNRSYNYREEANARFQASNPISIYLKDSNMLRPLNQNNLKI- } \\
\text { YGEEVLWGNNLNLAYEPKIGQQFFIKTLYSVQSDKIVREGDG- } \\
\text { ANYIDNFNFKSTNLNFI }\end{array}$ \\
\hline & $\underline{4}$ & WSOGGTDLANGYRRLGNNPDGTR \\
\hline & $\underline{5}$ & REIAQRNFTGSDRDVIYPIPGEVIYNPLAYANGNRKIYER \\
\hline & $\underline{6}$ & SWNGFNTSYGCKTNSEEERLLLVRANICDAT \\
\hline & 9 & DSKIYGLISTGQVDPLSTYAAYSPTTLNRPLQGOSD \\
\hline \multirow[t]{2}{*}{$\underline{\mathrm{LIC} 10964}$} & $\underline{3}$ & DLLDNPNENPGATVSQKLLHEKSHFHSFFIFSAGNLELDFSYQR \\
\hline & $\underline{8}$ & IQNFIYAASIAQIDLDSGLPKYEYKQGN \\
\hline \multirow[t]{2}{*}{$\underline{\mathrm{LIC} 12374}$} & $\underline{2}$ & DQNFSYKNDHGTVVLNTLDDTIDRRKNAS \\
\hline & $\underline{6}$ & FPSEEPWYRRQDPLSGDIK \\
\hline
\end{tabular}

noguchii showed the highest conservation with the L. interrogans TBDRs, with 7/9 fragments presenting more than $95 \%$ identity.

\section{BCG-vectored rTBDRchi protects hamsters against $L$. interrogans challenge}

The rTBDRchi CDS was synthesized and cloned into 
pUP500/ $\mathrm{P}_{\mathrm{pAN}}$ vectors (Oliveira et al. 2019b), allowing its heterologous expression in M. bovis BCG, as demonstrated by immunoblot (Online Resource 1, Figure S2). Subcutaneous immunization of hamsters with $10^{6}$ live rBCG:TBDRchi mycobacteria elicited $100 \%$ protection against homologous L. interrogans challenge in two independent experiments $(20 / 20$ animals, $p<0.001)$ with animals surviving for 30 days post-challenge (Table 2, Fig. 2). In contrast, all animals inoculated with wild-type BCG Pasteur (26/26) developed endpoint criteria, with clinical signs and weight-loss observed for all animals 13-15 days post-challenge (Fig. 2). Leptospira burden in kidneys was evaluated by culture and lipL32 qPCR using tissues from animals immunized with rBCG-TBDRchi or from the control groups (Table 2). Cultures from macerated kidneys from the negative control group (BCG Pasteur) were positive for leptospires following two weeks of incubation. No growth was detected after 8 weeks in the cultures from the rBCG:TBDRchi and bacterin groups. Similarly, lipL32 qPCR analysis was negative for leptospiral DNA in the kidneys of animals vaccinated with recombinant BCG (Table 2). The leptospiral burden in the negative control group ranged from $1.08 \times 10^{1}$ to $6.97 \times 10^{4}$ genome equivalents per $\mu \mathrm{g}$ of total extracted DNA, with an average of $5.03 \times 10^{3}$ (Table 2, Online Resource 1 Table S5).

\section{rBCG:TBDRchi elicits a humoral immune response in hamsters}

The immunogenicity of rBCG:TBDRchi was evaluated by indirect ELISA using rTBDRchi as the antigen. Hamsters vaccinated with $\mathrm{BBCG}$ :TBDRchi produced a significant antirTBDRchi IgG response $(p<0.05)$, detected 30 days after the second dose (Fig. 3a), demonstrating the potential of rBCG:TBDRchi to promote a humoral immune response. In contrast, hamsters injected with BCG-Pasteur were negative for rTBDRchi IgG in the same ELISA. The presence of antibodies against each leptospiral TBDR in immunized hamsters was assessed by Western blotting (WB). Sera obtained

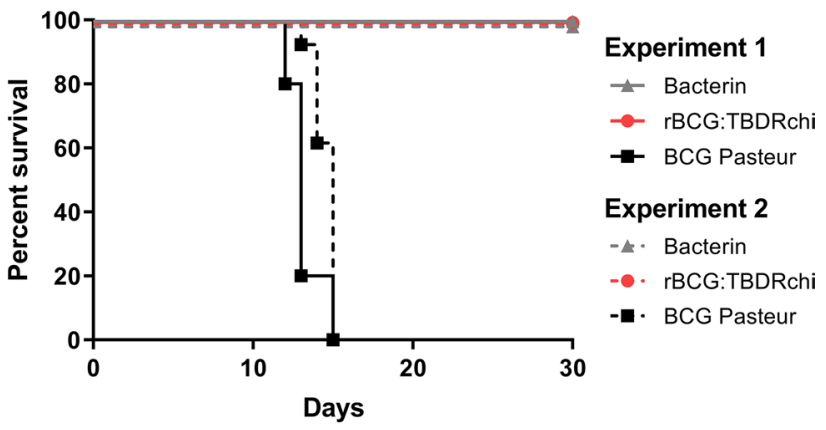

Fig. 2 Protection against lethal leptospirosis elicited by immunization with rBCG:TBDRchi. a Survival data from vaccinated hamster after challenge with an endpoint dose of L. interrogans sv. Copenhageni st. Fiocruz L1-130 in two independent experiments. All animals that were immunized subcutaneously with $10^{6}$ recombinant BCG (rBCG:TBDRchi, $n=10$ ) or $10^{7}$ leptospires (bacterin, $n=4$ ) survived $(p<0.001)$. Animals from the negative control group (BCG Pasteur, $n=13$ per experiment) reached endpoint criteria for euthanasia between days $12-15$ post-infection

from hamsters after immunization with rBCG:TBDRchi (1:200) detected two proteins in L. interrogans lysates, with apparent molecular weight of $\sim 100$ and $\sim 85 \mathrm{kDa}$, likely corresponding to LIC10896 $(99 \mathrm{kDa})$ and LIC10964 $(87 \mathrm{kDa})$ (Fig. 3b).

\section{Discussion}

Predicted surface-exposed fragments from three leptospiral TonB-dependent receptors were used in this work to construct the chimeric protein rTBDRchi. L. interrogans proteins LIC10896, LIC10964, and LIC12374 are putative TBDRs homologs (Nascimento et al. 2004; Louvel et al. 2006; Grassmann et al. 2017b). These observations were further supported in the current study; the 3D models of LIC10896, LIC10964, and LIC12374 presented high similarity to previously described TBDRs from other bacteria
Table 2 Protection against lethal leptospirosis elicited by immunization with rBCG:TBDRchi. Syrian hamsters were immunized with $10^{6}$ cells of BCG Pasteur wild-type or recombinant BCG:TBDRchi. Thirty days after the second dose, animals were injected intraperito- neally with $5 \times$ median endpoint dose (ED50) of L. interrogans serovar Copenhageni st. Fiocruz L1-130. Data from two independent experiments are presented

\begin{tabular}{lllll}
\hline Vaccine & \% Protection ${ }^{\mathrm{a}}$ & $p$ Value $^{\mathrm{b}}$ & \multicolumn{2}{l}{ Leptospires in kidneys $^{\mathrm{c}}$} \\
\cline { 4 - 5 } & & & \% Positive Culture & $0(0 / 20)$ \\
rBCG:TBDRchi & $100(20 / 20)$ & $<0.001$ & $0(0 / 20)$ & $100(26 / 26)$ \\
BCG Pasteur & $0(0 / 26)$ & $n s$ & $100(26 / 26)$ & $0(0 / 8)$ \\
Bacterin & $100(8 / 8)$ & $<0.001$ & $0(0 / 8)$ & Positive qPCR \\
\hline
\end{tabular}

${ }^{a}$ In parenthesis: survivors/total hamsters from two independent experiments

${ }^{\mathrm{b}} p$ Values were calculated by Fisher's exact test (two-tailed) for each experiment

${ }^{\mathrm{c}}$ In parenthesis: number of positive kidneys/total samples 


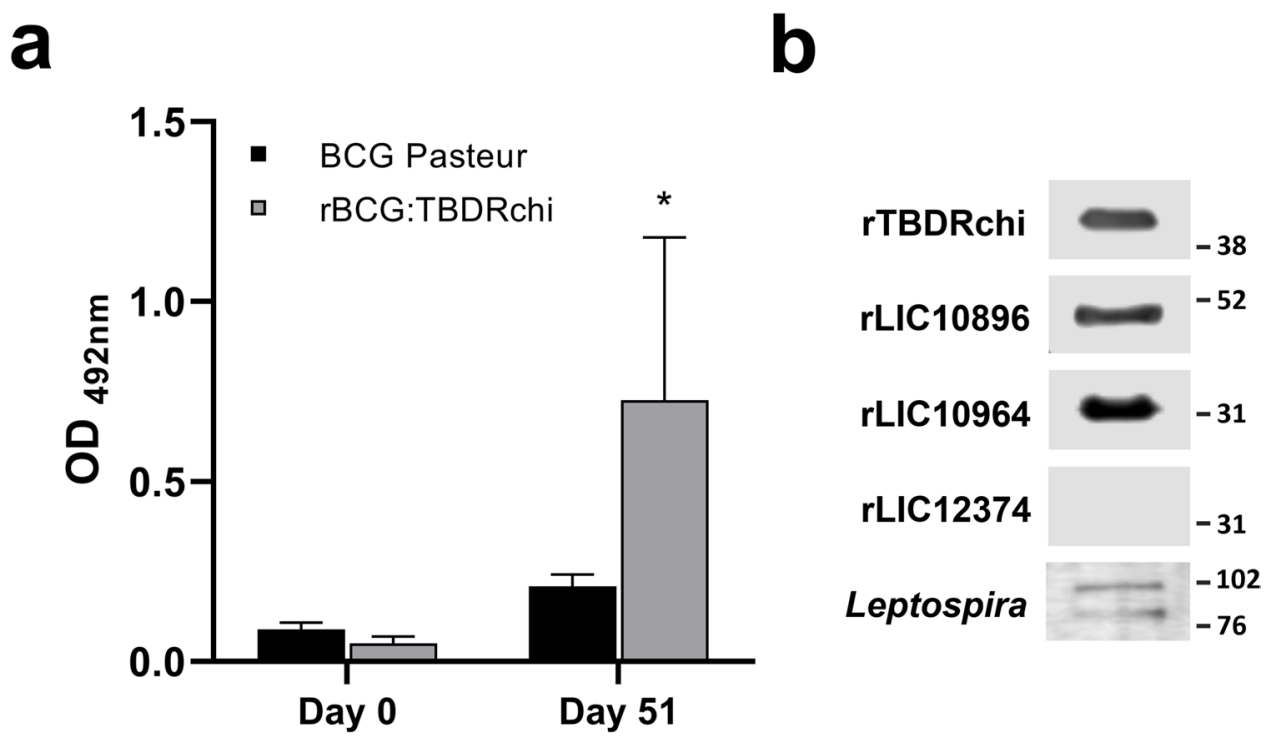

Fig. 3 IgG antibody response in hamsters immunized with rBCG:TBDRchi evaluated by ELISA and immunoblot. a ELISA plates were coated with $1 \mu \mathrm{g}$ of rTBDRchi. Sera from hamsters immunized with BCG Pasteur (negative control) and rBCG:TBDRchi collected at day 0 (pre-immune) and 51 (pre-challenge) were evaluated at 1:50 dilution, in triplicate, with an anti-hamster IgG used as secondary antibody. Results are presented as mean absorb-

(Online Resource 1, Table S3). Among the three structural models, LIC12374 is the model with the highest RMSD when aligned to its closest structural analog in PDB, the cobalamin transporter BtuB from E. coli (Chimento et al. 2003). Previously reported as a leptospiral FecA ortholog, LIC10896 was modeled with high structural similarity to the FpvA pyoverdine-Fe transporter from Pseudomonas aeruginosa (Cobessi et al. 2005). Similarly, LIC10964 was previously associated with hemin metabolism in Leptospira spp., and our 3D model was highly similar to the $N$. meningitidis zinc transporter, ZnuD (Calmettes et al. 2015). Identification of specific functions for TBDRs through structural modelling is difficult and should be determined experimentally. Independently of the specific substrate for a TBDR, the 22 transmembrane $\beta$-strand barrel conformation is highly conserved among known TBDR structures, supporting our approach for the identification of surface-exposed regions.

While LIC10896 and LIC12374 have orthologs conserved in all pathogenic and saprophyte species, LIC10964 is present only in pathogenic species, as previously noted (Grassmann et al. 2017a). Host-adapted leptospires, cultivated within dialysis membrane chambers (DMCs), expressed higher levels of LIC10964 compared to those cultivated in vitro (Caimano et al. 2014), suggesting a potential role during infection, in agreement with its predicted function as hemin transporter. The high level of sequence conservation of TBDRs across pathogenic leptospires makes this class of proteins an attractive target for development of ance \pm standard deviation. Significant differences were determined by one-way ANOVA, followed by Tukey multiple comparison. Asterisk indicates a significance difference $(p<0.05)$ to other groups. b Immunoblot with rTBDRchi, recombinant TBDR proteins and Leptospira lysate using sera obtained from hamsters after immunization with rBCG:TBDRchi (1:200). Humoral immune response was primarily direct against two of the leptospiral TBDRs, LIC10896 and LIC10964

cross-protective leptospirosis vaccines. In agreement with this notion, the TBDR TbpA from Haemophilus parasuis was able to induce a cross-protective immune response against three different serovars in a guinea pig model of Glasser's disease, with protection varying between 60 and $80 \%$ (Huang et al. 2013). Similarly, hyperimmune sera generated in mice and guinea pigs against $N$. meningitidis outer membrane vesicles overexpressing the TBDR ZnuD presented a neutralizing effect on 14 different $N$. meningitidis strains in vitro (Hubert et al. 2013). Although we did not evaluate rBCG:TBDRchi in a heterologous challenge, all of the epitopes in the recombinant vaccine antigen were highly conserved in other pathogenic Leptospira spp. (Online Resource 3), enhancing the possibility of heterologous protection after immunization, a requisite for a universal leptospiral vaccine (Grassmann et al. 2017b).

Multi-epitope constructs based on different proteins have been reported for leptospirosis diagnosis and vaccine development. Lin et al. (2016) evaluated a recombinant protein containing four repeats of $\mathrm{T}$ and $\mathrm{B}$ cell epitopes in the leptospiral proteins OmpL1, LipL32, and LipL21. That chimeric protein also stimulated significant protection $(4 / 5)$ in a guinea pig model of leptospirosis and induced a cross-reactive antibody response against various $L$. interrogans serogroups. Fernandes et al. (2017) designed a chimeric protein based on sequences from five leptospiral proteins; however, this construction failed to induce significant protection in hamsters, where $50 \%$ survived, 
despite the presence of epitopes from LigA, a well-known protective antigen (Yan et al. 2009; Coutinho et al. 2011). To the best of our knowledge, this study pioneered the use of protein structural information on leptospiral $\beta b$-OMPs to construct a recombinant molecule for use in an experimental vaccine against leptospirosis. In the current study, the humoral response generated by rBCG:TBDRchi was predominantly directed against two (LIC10896 and LIC10964) of the three proteins used in the antigen construction (Fig. 3b). However, this does not rule out additional antibodies against LIC12374 that were below the limit of detection for the Western blot and might have contributed to the protection observed, given the presumable importance of LIC12374 epitopes in rTBDRchi. Different antigen presentation strategies for the selected epitopes should be assessed in the future, towards the induction of strong antibody responses against all epitopes in rTBDRchi. Our approach for designing rTBDRchi antigen proved to be successful, given the protection we observed in the hamster model of acute leptospirosis.

Recombinant BCG vaccines have been shown to elicit both cellular and humoral immunity (Oliveira et al. 2017). In the current study, immunization with rBGG:TBDRchi induced a robust humoral immune responses against highly conserved surface-exposed epitopes from leptospiral TBDRs, as demonstrated by ELISA and Western blot. In contrast, in our previous study, we were unable to detect significant levels of antibodies by ELISA, in sera from animals immunized with a chimeric antigen containing fragments from the leptospiral lipoproteins LipL32, LigAni, and LemA (Oliveira et al. 2019a; Dorneles et al. 2020). The rationale of using epitope-rich fragments may have contributed to the induction of humoral response obtained by rBGG:TBDRchi. As an extracellular bacterium, clearance of leptospires by the immune system seems to be related to bacterial opsonization followed by phagocytosis (Gomes-Solecki et al. 2017; Santecchia et al. 2020). Different studies have shown that opsonization is a requirement for efficient bactericidal activity of macrophages in leptospirosis (Johnson and Muschel 1966; Banfi et al. 1982; Wang et al. 1984; Santecchia et al. 2020). Studies on phagocytosis without opsonization showed that pathogenic leptospires have several mechanisms to survive and escape killing by phagocytic cells (Zhang et al. 2012; $\mathrm{Hu}$ et al. 2013; Zhao et al. 2013; Toma et al. 2014). It is accepted that opsonization with immune serum enhances Leptospira uptake and killing by human macrophages; the role of phagocytic cells during infection was recently reviewed (Santecchia et al. 2020). These observations support the importance of the humoral response promoted by rBCG:TBDRchi immunization in the clearance of virulent leptospires during early stages of infection.
The apparent absence of leptospires in the kidneys of rBCG:TBDRchi-immunized hamsters agrees with previous studies using BCG vectorized vaccines against leptospirosis (Oliveira et al. 2019a; Dorneles et al. 2020). In contrast, several subunit vaccines against leptospirosis have failed to induce sterilizing immunity (Felix et al. 2020). It is believed that a robust and effective cell-mediated response may contribute to limit the initial tissue colonization by Leptospira spp. and contribute towards bacterial clearance (Zuerner 2015; Santecchia et al. 2019). A BCG-induced trained immunity has been shown to decrease host susceptibility against different pathogens (Covián et al. 2019). Trained immunity is a proposed term to describe the enhancement of a non-specific immune response generated against unrelated infections after previous exposure to microbial fragments (Netea et al. 2011). This phenomenon has been observed after inoculation of mycobacterial components such as muramyl dipeptide, complete Freund's adjuvant, and live BCG (Netea et al. 2011; Kleinnijenhuis et al. 2012). BCG vaccination increases IFN- $\gamma$ production and other monocyte-derived cytokines, such as tumor necrosis factors (TNF) and IL-1 $\beta$, through an NOD2 pathway (Kleinnijenhuis et al. 2012). Interestingly, the effects of trained immunity on leptospirosis were previously described using CL429, a TLR2, and NOD2 pathway synthetic agonist (Santecchia et al. 2019). CL429-trained macrophages produced significantly more pro-inflammatory cytokines in response to pathogenic Leptospira than naïve macrophages, independently of $\mathrm{B}$ and $\mathrm{T}$ cells. The same trained macrophages also produced increased amounts of nitric oxide (Santecchia et al. 2019), a potent antimicrobial compound previously related to leptospiral clearance (Prêtre et al. 2011). Unfortunately, the lack of immunological reagents to characterize cellular immunity in the hamster model for acute leptospirosis limited in-depth analyses of cellular responses elicited by rBCG:TBDRchi immunization.

In conclusion, using a structural vaccinology approach, we designed a chimeric antigen that included predicted surface-exposed $\mathrm{B}$ and $\mathrm{T}$ cell epitopes from $L$. interrogans TBDRs. We used a BCG delivery system targeting robust immune response, resulting in production of a humoral immune response specific to the selected epitopes in leptospires. This vaccine formulation induced a protective and sterilizing immune response in the hamster model of lethal leptospirosis following a homologous challenge with $L$. interrogans.

Supplementary Information The online version contains supplementary material available at https://doi.org/10.1007/s00253-021-11726-9.

Acknowledgements We are grateful to our lab technician Michele dos Santos and the UFPel's animal facility staff for the assistance provided during this study. 
Author contribution EB, JD, AG, AM, TO, and OD contributed to conceive and design the study. $\mathrm{EB}, \mathrm{JD}, \mathrm{AH}$, and $\mathrm{AM}$ performed research throughout the work. JD, AH, AM, and ASN performed animal experimentations. EB, AH, and JD analyzed the data. EB, AG, and AM wrote the paper. All authors contributed for manuscript review.

Funding Execution of this work was possible thanks to the support of CAPES, CNPq, and FAPERGS through grants and scholarships. This study was financed in part by the Coordenação de Aperfeiçoamento de Pessoal de Nível Superior-Brasil (CAPES)—Finance Code 001.

Data availability Leptospira interrogans Fiocruz L1-130 strain is available as described in the Fiocruz/CLEP collection (WDCM 1012). Raw data from epitope mapping are available as Online Resource (ESM_3). Any additional data and materials used in this article are available upon request.

\section{Declarations}

Ethics approval All procedures involving manipulations of animals were approved by the Ethics Committee in Animal Experimentation (CEEA) of Universidade Federal de Pelotas (UFPel) under protocol number 4646-2015. CEEA-UFPel is accredited by the Brazilian National Council for Animal Experimentation Control (CONCEA).

Conflict of interest The authors declare no competing interests.

\section{References}

Adler B (2015) Vaccines against leptospirosis. Curr Top Microbiol Immunol 387:251-272. https://doi.org/10.1007/978-3-662-45059$8 \_10$

Alteri CJ, Hagan EC, Sivick KE, Smith SN, Mobley HLT (2009) Mucosal immunization with iron receptor antigens protects against urinary tract infection. PLoS Pathogen 5:e1000586. https://doi.org/10.1371/journal.ppat.1000586

Banfi E, Cinco M, Bellini M, Soranzo MR (1982) The role of antibodies and serum complement in the interaction between macrophages and leptospires. J Gen Microbiol 128:813-816. https:// doi.org/10.1099/00221287-128-4-813

Caimano MJ, Sivasankaran SK, Allard A, Hurley D, Hokamp K, Grassmann A, Hinton JCD, Nally JE (2014) A model system for studying the transcriptomic and physiological changes associated with mammalian host-adaptation by Leptospira interrogans Serovar Copenhageni. PLoS Pathog 10:e1004004. https://doi.org/10.1371/ journal.ppat. 1004004

Calmettes C, Ing C, Buckwalter CM, el Bakkouri M, Lai CC, Moraes TF, Pogoutse A, Gray-owen SD (2015) The molecular mechanism of zinc acquisition by the neisserial outer-membrane transporter ZnuD. Nat Commun 6:1-11. https://doi.org/10.1038/ncomms8996

Chimento DP, Kadner RJ, Wiener MC (2003) The Escherichia coli outer membrane cobalamin transporter BtuB: structural analysis of calcium and substrate binding, and identification of orthologous transporters by sequence/structure conservation. J Mol Biol 332:999-1014. https://doi.org/10.1016/j.jmb.2003.07.005

Cobessi D, Celia H, Folschweiller N, Schalk IJ, Abdallah MA, Pattus F (2005) The crystal structure of the pyoverdine outer membrane receptor FpvA from Pseudomonas aeruginosa at 3.6 A Resolution. J Mol Biol 347:121-134. https://doi.org/10.1016/j.jmb.2005. 01.021

Costa F, Hagan JE, Calcagno J, Kane M, Torgerson P, Martinez-silveira MS, Stein C, Abela-ridder B, Ko AI (2015) Global morbidity and mortality of leptospirosis : a systematic review. PLoS Negl Trop Dis 9:e0003898. https://doi.org/10.1371/journal.pntd.0003898

Coutinho ML, Choy HA, Kelley MM, Matsunaga J, Babbitt JT, Lewis MS, Aleixo JAG, Haake DA (2011) A LigA three-domain region protects hamsters from lethal infection by Leptospira interrogans. PLoS Negl Trop Dis 5:1-10. https://doi.org/10.1371/journal.pntd. 0001422

Covián C, Fernández-Fierro A, Retamal-Díaz A, Díaz FE, Vasquez AE, Lay MK, Riedel CA, González PA, Bueno SM, Kalergis AM (2019) BCG-induced cross-protection and development of trained immunity: implication for vaccine design. Front Immunol 10:1-14. https://doi.org/10.3389/fimmu.2019.02806

da Cunha CEP, Bettin EB, Bakry AFAAY, Seixas Neto ACP, Amaral MG, Dellagostin OA (2019) Evaluation of different strategies to promote a protective immune response against leptospirosis using a recombinant LigA and LigB chimera. Vaccine 37:1844-1852. https://doi.org/10.1016/j.vaccine.2019.02.010

Dellagostin OA, Grassmann AA, Rizzi C, Schuch RA, Jorge S, Oliveira TL, Mcbride AJA, Hartwig DD (2017) Reverse vaccinology: an approach for identifying leptospiral vaccine candidates. Int J Mol Sci 18:158. https://doi.org/10.3390/ijms18010158

Dockrell HM, Smith SG (2017) What have we learnt about BCG vaccination in the last 20 years? Frontiers in Immunology $8 \mathrm{https}: / /$ doi.org/10.3389/fimmu.2017.01134

Dorneles J, Bonemann A, Clair A, Seixas P, Rizzi C, Burlamarque É, Silva A, Caetano C, Castro D, Gevehr C, Larré T, Antonio O (2020) Protection against leptospirosis conferred by Mycobacterium bovis $\mathrm{BCG}$ expressing antigens from Leptospira interrogans. Vaccine 38:8136-8144. https://doi.org/10.1016/j.vaccine.2020. 10.086

Edgar RC, Drive RM, Valley M (2004) MUSCLE: multiple sequence alignment with high accuracy and high throughput. Nucleic Acids Res 32:1792-1797. https://doi.org/10.1093/nar/gkh340

Ellis WA (2015) Animal leptospirosis. Curr Top Microbiol Immunol 387:99-137. https://doi.org/10.1007/978-3-662-45059-8_6

Faine S (1959) Iron as a growth requirement for pathogenic Leptospira. J Gen Microbiol 20:246-251. https://doi.org/10.1099/00221 287-20-2-246

Felix CR, Siedler BS, Barbosa LN, Timm GR, McFadden J, McBride AJA (2020) An overview of human leptospirosis vaccine design and future perspectives. Expert Opin Drug Discov 15:179-188. https://doi.org/10.1080/17460441.2020.1694508

Fernandes LGV, Teixeira AF, Filho AFS, Souza GO, Vasconcellos SA, Heinemann MB, Romero EC, Nascimento ALTO (2017) Immune response and protective profile elicited by a multi-epitope chimeric protein derived from Leptospira interrogans. Int J Infect Dis 57:61-69. https://doi.org/10.1016/j.ijid.2017.01.032

Fouts DE, Matthias MA, Adhikarla H, Adler B, Amorim-Santos L, Berg DE, Bulach D, Buschiazzo A, Chang YF, Galloway RL, Haake DA, Haft DH, Hartskeerl R, Ko AI, Levett PN, Matsunaga J, Mechaly AE, Monk JM, Nascimento ALT, Nelson KE, Palsson B, Peacock SJ, Picardeau M, Ricaldi JN, Thaipandungpanit J, Wunder EA, Yang XF, Zhang JJ, Vinetz JM, Derrick E, Michael A, Fouts DE, Matthias MA, Adhikarla H, Adler B, Amorim- L (2016) What makes a bacterial species pathogenic?: Comparative genomic analysis of the genus Leptospira. PLoS Negl Trop Dis 10:1-57. https://doi.org/10.1371/journal.pntd.0004403

Gomes-Solecki M, Santecchia I, Werts C (2017) Animal models of leptospirosis: of mice and hamsters. Frontiers in Immunology 8https://doi.org/10.3389/fimmu.2017.00058

Grassmann AA, Kremer FS, dos Santos JC, Souza JD, da Pinto LS, McBride AJA (2017a) Discovery of novel leptospirosis vaccine candidates using reverse and structural vaccinology. Frontiers in Immunology 8:463. https://doi.org/10.3389/fimmu.2017.00463

Grassmann AA, Souza JD, John A, Mcbride AJA, John A, Mcbride AJA (2017b) A universal vaccine against leptospirosis: are we 
going in the right direction? Front Immunol 8:1-11. https://doi. org/10.3389/fimmu.2017.00256

Gröschel MI, Sayes F, Shin SJ, Frigui W, Pawlik A, Orgeur M, Canetti R, Honoré N, Simeone R, van der Werf TS, Bitter W, Cho SN, Majlessi L, Brosch R (2017) Recombinant BCG Expressing ESX-1 of Mycobacterium marinum Combines Low Virulence with Cytosolic Immune Signaling and Improved TB Protection. Cell Rep 18:2752-2765. https://doi.org/10.1016/j.celrep.2017. 02.057

Groshong AM, Grassmann AA, Luthra A, McLain MA, Provatas AA, Radolf JD, Caimano MJ (2021) PlzA is a bifunctional c-di-GMP biosensor that promotes tick and mammalian host adaptation of Borrelia burgdorferi. PLoS Pathog 17:1-31. https://doi.org/10. 1371/journal.ppat.1009725

Haake DA, Levett PN (2015) Leptospirosis in humans. Curr Top Microbiol Immunol 387:65-97. https://doi.org/10.1007/ 978-3-662-45059-8

Hu W, Ge Y, Ojcius DM, Sun D, Dong H, Yang XF, Yan J (2013) p53 controls apoptosis in leptospira-infected macrophages. Cell Microbiol 15:1642-1659. https://doi.org/10.1111/cmi.12141

Hu Y, Dang W, Sun L (2012) A TonB-dependent outer membrane receptor of Pseudomonas fluorescens: virulence and vaccine potential. Arch Microbiol 194:795-802. https://doi.org/10.1007/ s00203-012-0812-3

Huang X, Li Y, Fu Y, Ji Y, Lian K, Zheng H, Wei J, Cai X (2013) Cross-Protective Efficacy of Recombinant Transferrin-Binding Protein A of Haemophilus parasuis in Guinea Pigs. Clin Vaccine Immunol 20:912-919. https://doi.org/10.1128/CVI.00621-12

Hubert K, Devos N, Mordhorst I, Tans C, Baudoux G, Feron C, Goraj K, Tommassen J, Vogel U, Poolman JT (2013) ZnuD, a potential candidate for a simple and universal Neisseria meningitidis Vaccine. Infect Immun 81:1915-1927. https://doi.org/10.1128/IAI. 01312-12

Johnson RC, Muschel LH (1966) Antileptospiral activity of serum. I. Normal and immune serum. J Bacteriol 91:1403-1409. https:// doi.org/10.1128/jb.91.4.1403-1409.1966

Kleinnijenhuis J, Quintin J, Preijers F, Joosten LAB, Ifrim DC, Saeed S, Jacobs C, Van Loenhout J, De Jong D, Hendrik S, Xavier RJ, Van Der Meer JWM, Van Crevel R, Netea MG (2012) Bacille Calmette-Guérin induces NOD2-dependent nonspecific protection from reinfection via epigenetic reprogramming of monocytes. Proc Natl Acad Sci USA 109:17537-17542. https://doi.org/10. 1073/pnas.1202870109

Larsen JEP, Lund O, Nielsen M (2006) Improved method for predicting linear B-cell epitopes. Immunome Research 2:2. https://doi.org/ 10.1186/1745-7580-2-2

Lin X, Xiao G, Luo D, Kong L, Chen X, Sun D, Yan J (2016) Chimeric epitope vaccine against Leptospira interrogans infection and induced specific immunity in guinea pigs. BMC Microbiology 16https://doi.org/10.1186/s12866-016-0852-y

Liu J, Ma Q, Yang F, Zhu R, Gu J, Sun C, Feng X, Du C, Langford PR, Han W, Yang J, Lei L (2017) B cell cross-epitope of Propionibacterium acnes and Actinobacillus pleuropneumonia selected by phage display library can efficiently protect from Actinobacillus pleuropneumonia infection. Vet Microbiol 205:14-21. https://doi. org/10.1016/j.vetmic.2017.04.026

Lobo N, Brooks NA, Zlotta AR, Cirillo JD, Boorjian S, Black PC, Meeks JJ, Bivalacqua TJ, Gontero P, Steinberg GD, McConkey D, Babjuk M, Alfred Witjes J, Kamat AM (2021) 100 years of Bacillus Calmette-Guérin immunotherapy: from cattle to COVID-19. Nat Rev Urol 18:611-622. https://doi.org/10.1038/ s41585-021-00481-1

Lomize MA, Pogozheva ID, Joo H, Mosberg HI, Lomize AL (2012) OPM database and PPM web server: Resources for positioning of proteins in membranes. Nucleic Acids Res 40:370-376. https:// doi.org/10.1093/nar/gkr703

Louvel H, Bommezzadri S, Zidane N, Boursaux-Eude C, Creno S, Magnier A, Rouy Z, Médigue C, Saint Girons I, Bouchier C, Picardeau $M$ (2006) Comparative and functional genomic analyses of iron transport and regulation in Leptospira spp. J Bacteriol 188:7893-7904. https://doi.org/10.1128/JB.00711-06

Nascimento ALTO, Ko AI, Martins EAL, Monteiro-Vitorello CB, Ho PL, Haake DA, Verjovski-Almeida S, Hartskeerl RA, Marques MV, Oliveira MC, Menck CFM, Leite LCC, Carrer H, Coutinho LL, Degrave WM, Dellagostin OA, El-Dorry H, Ferro ES, Ferro MIT, Furlan LR, Gamberini M, Giglioti EA, Góes-Neto A, Goldman GH, Goldman MHS, Harakava R, Jerônimo SMB, Junqueira-De-Azevedo ILM, Kimura ET, Kuramae EE, Lemos EGM, Lemos MVF, Marino CL, Nunes LR, De Oliveira RC, Pereira GG, Reis MS, Schriefer A, Siqueira WJ, Sommer P, Tsai SM, Simpson AJG, Ferro JA, Camargo LEA, Kitajima JP, Setubal JC, Van Sluys MA (2004) Comparative genomics of two Leptospira interrogans serovars reveals novel insights into physiology and pathogenesis. J Bacteriol 186:2164-2172. https://doi.org/10.1128/ JB.186.7.2164-2172.2004

Netea MG, Quintin J, Van Der Meer JWM (2011) Trained immunity: a memory for innate host defense. Cell Host Microbe 9:355-361. https://doi.org/10.1016/j.chom.2011.04.006

Nielsen M, Lund O (2009) NN-align. An artificial neural networkbased alignment algorithm for MHC class II peptide binding prediction. BMC Bioinformatics 10:296. https://doi.org/10.1186/ 1471-2105-10-296

Noinaj N, Guillier M, Barnard TJ, Buchanan SK (2010) TonB-dependent transporters: regulation, structure, and function. Annu Rev Microbiol 43-60https://doi.org/10.1146/annurev.micro.112408. 134247

Nosrati M, Hajizade A, Nazarian S, Amani J, Namvar Vansofla A, Tarverdizadeh Y (2019) Designing a multi-epitope vaccine for cross-protection against Shigella spp: An immunoinformatics and structural vaccinology study. Mol Immunol 116:106-116. https:// doi.org/10.1016/j.molimm.2019.09.018

Oliveira TL, Rizzi C, da Cunha CEP, Dorneles J, Seixas Neto ACP, Amaral MG, Hartwig DD, Dellagostin OA (2019a) Recombinant BCG strains expressing chimeric proteins derived from Leptospira protect hamsters against leptospirosis. Vaccine 37:776-782. https://doi.org/10.1016/j.vaccine.2018.12.050

Oliveira TL, Rizzi C, Dellagostin OA (2017) Recombinant BCG vaccines: molecular features and their influence in the expression of foreign genes. Appl Microbiol Biotechnol 101:6865-6877. https:// doi.org/10.1007/s00253-017-8439-6

Oliveira TL, Stedman A, Rizzi C, Dorneles J, da Cunha CEP, Junior ASV, Dellagostin OA, McFadden J (2019b) A standardized BioBrick toolbox for the assembly of sequences in mycobacteria. Tuberculosis 119:101851. https://doi.org/10.1016/j.tube.2019.07. 002

Oyarzún P, Kobe B (2016) Recombinant and epitope-based vaccines on the road to the market and implications for vaccine design and production. Hum Vaccin Immunother 12:763-767. https://doi.org/ 10.1080/21645515.2015.1094595

Parish T, Stoker NG (1995) Electroporation of mycobacteria. Methods in Molecular Biology (clifton, NJ) 47:237-252. https://doi.org/10. 1385/0-89603-310-4:237

Parvizpour S, Pourseif MM, Razmara J, Rafi MA, Omidi Y (2020) Epitope-based vaccine design: a comprehensive overview of bioinformatics approaches. Drug Discovery Today 25:1034-1042. https://doi.org/10.1016/j.drudis.2020.03.006

Pettersen EF, Goddard TD, Huang CC, Couch GS, Greenblatt DM, Meng EC, Ferrin TE (2004) UCSF Chimera - a visualization 
system for exploratory research and analysis. J Comput Chem 1605-1612https://doi.org/10.1002/jcc.20084

Prêtre G, Olivera N, Cédola M, Haase S, Alberdi L, Brihuega B, Gómez RM (2011) Role of inducible nitric oxide synthase in the pathogenesis of experimental leptospirosis. Microb Pathog 51:203-208. https://doi.org/10.1016/j.micpath.2011.03.011

Ramos CRR, Abreu PAE, Nascimento ALTO, Ho PL (2004) A highcopy T7 Escherichia coli expression vector for the production of recombinant proteins with a minimal $\mathrm{N}$-terminal his-tagged fusion peptide. Braz J Med Biol Res 37:1103-1109. https://doi.org/10. 1590/S0100-879X2004000800001

Sanchez-trincado JL, Gomez-perosanz M, Reche PA (2017) Fundamentals and methods for T- and B-cell epitope prediction. Journal of Immunology Research 2017https://doi.org/10.1155/2017/ 2680160

Santecchia I, Ferrer F, Vieira ML, Mart R (2020) Phagocyte escape of Leptospira: the role of TLRs and NLRs. Frontiers in Immunology $11 \mathrm{https} / / /$ doi.org/10.3389/fimmu.2020.571816

Santecchia I, Vernel-Pauillac F, Rasid O, Quintin J, Gomes-Solecki M, Boneca IG, Werts C (2019) Innate immune memory through TLR2 and NOD2 contributes to the control of Leptospira interrogans infection. PLoS Pathog 15:1-26. https://doi.org/10.1371/ journal.ppat.1007811

Seixas F, Drawanz D, Maia G, Amaral M, Quevedo M (2007) Recombinant Mycobacterium bovis BCG expressing the LipL32 antigen of Leptospira interrogans protects hamsters from challenge. Vaccine 26:88-95. https://doi.org/10.1016/j.vaccine.2007.10.052

Shelton AN, Seth EC, Mok KC, Han AW, Jackson SN, Haft DR, Taga ME (2019) Uneven distribution of cobamide biosynthesis and dependence in bacteria predicted by comparative genomics. ISME J 13:789-804. https://doi.org/10.1038/s41396-018-0304-9

Soto JA, Gálvez NMS, Rivera CA, Palavecino CE, Céspedes PF, ReyJurado E, Bueno SM, Kalergis AM (2018) Recombinant BCG vaccines reduce pneumovirus-caused airway pathology by inducing protective humoral immunity. Front Immunol 9:2875. https:// doi.org/10.3389/fimmu.2018.02875

Toma C, Murray GL, Nohara T, Mizuyama M, Koizumi N, Adler B, Suzuki T (2014) Leptospiral outer membrane protein LMB216 is involved in enhancement of phagocytic uptake by macrophages. Cell Microbiol 16:1366-1377. https://doi.org/10.1111/cmi.12296

Trunz BB, Fine P, Dye C (2006) Effect of BCG vaccination on childhood tuberculous meningitis and miliary tuberculosis worldwide: a meta-analysis and assessment of cost-effectiveness. Lancet 367:1173-1180. https://doi.org/10.1016/S0140-6736(06)68507-3

Vincent AT, Schiettekatte O, Goarant C, Neela VK, Bernet E, Thibeaux R, Ismail N, Khalid MKNM, Amran F, Masuzawa T, Nakao R,
Korba AA, Bourhy P, Veyrier FJ, Picardeau M (2019) Revisiting the taxonomy and evolution of pathogenicity of the genus Leptospira through the prism of genomics. PLoS Neglected Tropical Diseases 13https://doi.org/10.1371/journal.pntd.0007270

Wang B, Sullivan JA, Sullivan GW, Mandell GL (1984) Role of specific antibody in interaction of leptospires with human monocytes and monocyte-derived macrophages. Infect Immun 46:809-813. https://doi.org/10.1128/iai.46.3.809-813.1984

Xiao J, Liu J, Bao C, Zhu R, Gu J, Sun C, Feng X, Du C, Han W, Li Y, Lei L (2020) Recombinant tandem epitope vaccination provides cross protection against Actinobacillus pleuropneumoniae challenge in mice. AMB Express 10:1-13. https://doi.org/10.1186/ s13568-020-01051-1

Yan W, Faisal SM, Mcdonough SP, Divers TJ, Barr SC, Chang C, Pan M, Chang Y (2009) Immunogenicity and protective efficacy of recombinant Leptospira immunoglobulin-like protein $\mathrm{B}(\mathrm{rLigB})$ in a hamster challenge model. Microbes Infect 11:230-237. https:// doi.org/10.1016/j.micinf.2008.11.008

Yang J, Zhang Y (2015) I-TASSER server: new development for protein structure and function predictions. Nucleic Acids Res 43:174181. https://doi.org/10.1093/nar/gkv342

Zhang L, Zhang C, Ojcius DM, Sun D, Zhao J, Lin X, Li L, Li L, Yan J (2012) The mammalian cell entry (Mce) protein of pathogenic Leptospira species is responsible for RGD motif-dependent infection of cells and animals. Mol Microbiol 83:1006-1023. https:// doi.org/10.1111/j.1365-2958.2012.07985.x

Zhang Y, Skolnick J (2005) TM-align: a protein structure alignment algorithm based on the TM-score. Nucleic Acids Res 33:23022309. https://doi.org/10.1093/nar/gki524

Zhao J, Chen H, Ojcius DM, Zhao X, Sun D, Ge Y, Zheng L, Lin X, Li L, Yan J (2013) Identification of Leptospira interrogans phospholipase $\mathrm{C}$ as a novel virulence factor responsible for intracellular free calcium ion elevation during macrophage death. PLoS ONE 8:e75652. https://doi.org/10.1371/journal.pone.0075652

Zuerner RL (2015) Host response to Leptospira infection. Curr Top Microbiol Immunol 387:223-250. https://doi.org/10.1007/ 978-3-662-45059-8_9

Publisher's Note Springer Nature remains neutral with regard to jurisdictional claims in published maps and institutional affiliations. 